\title{
Correction to: Clinical Chiari syndrome or anatomical Chiari malformation? A conundrum revisited
}

\section{Ekkehard M. Kasper ${ }^{1} \cdot$ Tiit Illimar Mathiesen ${ }^{2,3,4} \cdot$ Andreas K. Demetriades $^{5}$}

Published online: 19 May 2020

(C) Springer-Verlag GmbH Austria, part of Springer Nature 2020

\section{Correction to: Acta Neurochirurgica}

https://doi.org/10.1007/s00701-019-04175-x

An incorrect set of affiliations for two authors (Tiit Illimar Mathiesen and Andreas K. Demetriades).

An incorrect spelling for the name of one of the author Andreas K. Demetriades.

The original article has been corrected.

The online version of the original article can be found at https://doi.org/ 10.1007/s00701-019-04175-x

Ekkehard M. Kasper

kaspere@mcmaster.ca

1 Division of Neurosurgery, McMaster University Faculty of Health, Sciences and Hamilton Health Sciences Corporation, 237 Barton, Street East, Hamilton, ON L8L 2X2, Canada

2 Department of Neurosurgery, Rigshospitalet, Blegdamsvej 9, 2100 Copenhagen, Denmark

3 Institute of Clinical Medicine, University of Copenhagen, 2300 Copenhagen, Denmark

4 Department of Clinical Neuroscience, Karolinska Institutet, 171 76 Stockholm, Sweden

5 Department of Neurosurgery Western General Hospital Edinburgh, Edinburgh, UK 